\title{
Carboxylic acid derivatives of histone deacetylase inhibitors induce full length SMN2 transcripts: a promising target for spinal muscular atrophy therapeutics
}

Didem Dayangac-Erden¹, Gamze Bora-Tatar ${ }^{1}$, Sevim Dalkara², Ayhan S. Demir ${ }^{3}$, Hayat Erdem-Yurter ${ }^{1}$

1Department of Medical Biology, Faculty of Medicine, Hacettepe University, Ankara, Turkey

2Department of Pharmaceutical Chemistry, Faculty of Pharmacy, Hacettepe University, Ankara, Turkey

${ }^{3}$ Department of Chemistry, Middle East Technical University, Ankara, Turkey

Submitted: 12 August 2010

Accepted: 14 October 2010

Arch Med Sci 2011; 7, 2: 230-234

DOI: 10.5114/aoms.2011.22072

Copyright (c) 2011 Termedia \& Banach

\section{Abstract}

Introduction: Proximal spinal muscular atrophy (SMA) is a common autosomal recessively inherited neuromuscular disorder. It is caused by homozygous absence of the survival motor neuron 1 (SMN1) gene. SMN2, which modulates the severity of the disease, represents a major target for therapy. The aim of this study was to investigate whether SMN2 expression can be increased by caffeic acid, chlorogenic acid and curcumin, which are designed by modifications of the carboxylic acid class of histone deacetylase (HDAC) inhibitors.

Material and methods: Using quantitative real-time PCR, we analysed the levels of full-length SMN2 and $\triangle 7 S M N 2$ mRNA. We performed LDH cytotoxicity assay to analyse whether SMN2 activating concentrations of caffeic acid, chlorogenic acid and curcumin were cytotoxic to fibroblasts.

Results: We found that caffeic acid and curcumin were more efficient than chlorogenic acid and increased full-length SMN2 mRNA levels 1.5 and 1.7-fold, respectively. $\triangle 7 S M N 2$ mRNA levels were measured to investigate alternative splicing of exon 7. We also found that cytotoxicity was not observed at SMN2 activating concentrations.

Conclusions: Our data suggest that carboxylic acid derivatives including phenolic structure and symmetry could be a good candidate for SMA treatment.

Key words: histone deacetylase inhibitors, SMN2 gene, therapy.

\section{Introduction}

Autosomal recessive spinal muscular atrophy (SMA) is a leading genetic cause of childhood mortality. Patients develop symmetrical, proximal weakness resulting from neurogenic muscle atrophy. The disease is characterized by selective loss of large $\alpha$ motor neurons in the anterior horn of the spinal cord. Clinically, childhood-onset SMA is classified into three groups (type I: severe, type II: intermediate, type III: mild) according to age of onset and severity [1, 2]. All forms of SMA are caused by the homozygous deletion of the telomeric copy of the survival motor neuron (SMN1) gene on chromosome 5q13 [3-5], which codes the survival motor neuron (SMN) protein [6]. A second gene, centromeric copy of the SMN gene (SMN2) is also located at the same chromosomal region [3]. Because
Corresponding author:

Didem Dayangac-Erden, PhD

Hacettepe University

Faculty of Medicine

Department of Medical

Biology

Ankara, Turkey

Phone: 00903123052541

Fax: 00903123096060

E-mail:

didayan@hacettepe.edu.tr 
of a silent mutation (C to T) at position +6 in exon 7 , an exonic splicing enhancer (ESE) is destroyed and exon 7 skipping occurs in SMN2 pre-mRNA [7]. In contrast to SMN1, SMN2 only produces $10 \%$ fulllength transcripts (FL-SMN2). Ninety percent of SMN2 transcripts lack exon 7 ( $\triangle 7 S M N 2)$ and cannot compensate for the loss of SMN1 [8].

SMN2 is considered as the major target for a potential SMA therapy. Increasing SMN2 transcription and changing its splicing pattern are the most effective strategies [9]. Several histone deacetylase (HDAC) inhibitors which increase the expression of the SMN2 gene have been described [10-16]. The HDAC inhibitors stimulate acetylation of histones which can relax chromatin structure and enable binding of transcriptional machinery to target genes [17]. They are known to change the expression of $7-10 \%$ of genes [18]; but, how they activate SMN2 expression remains unknown.

Histone deacetylase inhibitors consist of a metal binding moiety, a capping group and a linker [19]. They are divided into hydroxamates, cyclic tetrapeptides, benzamides, electrophilic ketones and carboxylic acids [20]. The carboxylic acid class such as sodium butyrate, phenylbutyrate and valproic acid is the first investigated HDAC inhibitor group in the treatment of SMA. Their effect has been tested in SMA mouse models and treatment improved motor abilities and reduced neurodegeneration [21].

There are limited structure activity data for carboxylic acids. Therefore, in our previous study we developed new carboxylic acid derivatives by rational design and molecular modifications were made to the functional group, capping group and linker. In addition to the rational design approach, molecular docking which predicts inhibitor activity was used. After interpreting the results obtained from rational design and molecular docking tools, we found that among carboxylic acid derivatives, caffeic acid, chlorogenic acid and curcumin have the highest HDAC inhibition activity in a fluorimetric assay [22]. Since the SMN2 gene is present in all patients, it acts as a modifier gene. Therefore activating the SMN2 gene is a promising approach in SMA therapeutics. In this study, we investigated the effect of caffeic acid, chlorogenic acid and curcumin, which have HDAC inhibition activity, on SMN2 gene expression.

\section{Material and methods}

\section{Cell culture and treatment}

Human fibroblast cell line (Coriell Cell Repository, GM03813, 3 years old SMA type I) were cultured in DMEM with $10 \%$ FCS, $1 \%$ penicillin/streptomycin, $1 \%$ L-glutamine. $5 \times 10^{5}$ cells were treated with caffeic acid ( $20 \mu \mathrm{M}, 50 \mu \mathrm{M}, 100 \mu \mathrm{M})$, chlorogenic acid $(20 \mu \mathrm{M}, 50 \mu \mathrm{M}, 100 \mu \mathrm{M})$ and curcumin (12.5 $\mu \mathrm{M}, 20 \mu \mathrm{M}, 25 \mu \mathrm{M}, 50 \mu \mathrm{M}, 100 \mu \mathrm{M})$ for $24 \mathrm{~h}$. The concentrations used for the three derivatives were chosen according to the activity of known carboxylic acids $[23,24]$. For each experiment complete medium was added as a control.

\section{Quantitative analysis of FL-SMN2 and SMN2 27 transcripts}

Total RNA was isolated by RNeasy Mini Kit (Qiagen) and the absorbance was detected by NanoDrop ND-1000. 450 ng of RNA was converted to cDNA by Quantitect RT (Qiagen). Real-time RT-PCR was performed in triplicate by the iQ5 RealTime PCR Detection System (Biorad Lab). $25 \mu \mathrm{l}$ realtime RT-PCR reaction contained $0.096 \mu \mathrm{M}$ probe, $0.9 \mu \mathrm{M}$ of each primer and $12.5 \mu \mathrm{l}$ of TaqMan Universal Master Mix (Applied Biosystems). The conditions were $10 \mathrm{~min}$ at $95^{\circ} \mathrm{C}$, and 40 cycles of $15 \mathrm{~s}$ at $95^{\circ} \mathrm{C}$ and $1 \mathrm{~min}$ at $60^{\circ} \mathrm{C}$. The sequences of primers and TaqMan probes were published by Andreassi et al. [11]. The levels of SMN2 transcripts were quantified by $2^{-\Delta \Delta C t}$ method and normalized to GAPDH and $\beta$-actin (Applied Biosystems). The untreated sample was used as a calibrator. The experiments were repeated twice.

\section{Cytotoxicity assay}

Cytotoxicity of compounds was measured using LDH Cytotoxicity Assay Kit II (Biovision). $4 \times 10^{4}$ cells were plated in triplicate and incubated at $5 \% \mathrm{CO}_{2}$, $37^{\circ} \mathrm{C}$ for $24 \mathrm{~h}$. Untreated cells were used as low control and cells treated with lysis solution served as high control. Cytotoxicity was determined after $24 \mathrm{~h}$ of compound treatment. Following $30 \mathrm{~min}$ of incubation at room temperature, absorbance at $450 \mathrm{~nm}$ (reference wavelength was $650 \mathrm{~nm}$ ) was measured to determine LDH activity. Percentage of cytotoxicity was calculated as the ratio of test sample's absorbance to high control's absorbance.

\section{Results}

To determine the effect of carboxylic acid derivatives on SMN2 expression and splicing, the SMA fibroblast cell line was treated with caffeic acid $(20 \mu \mathrm{M}, 50 \mu \mathrm{M}, 100 \mu \mathrm{M})$, chlorogenic acid $(20 \mu \mathrm{M}$, $50 \mu \mathrm{M}, 100 \mu \mathrm{M})$ and curcumin $(12.5 \mu \mathrm{M}, 20 \mu \mathrm{M}$, $25 \mu \mathrm{M}, 50 \mu \mathrm{M}, 100 \mu \mathrm{M})$ for $24 \mathrm{~h}$. The levels of SMN2 mRNA were determined by real-time RT-PCR. All three compounds showed an increase in FL-SMN2 mRNA levels dose dependently (Figure 1). The analysis showed that $20 \mu \mathrm{M}$ caffeic acid increased FL-SMN2 1.5-fold (Figure 2A). After treatment with chlorogenic acid FL-SMN2 showed a minor increase at $50 \mu \mathrm{M}$ which was 1.3 -fold. However, this increase was not statistically significant compared to the untreated sample 


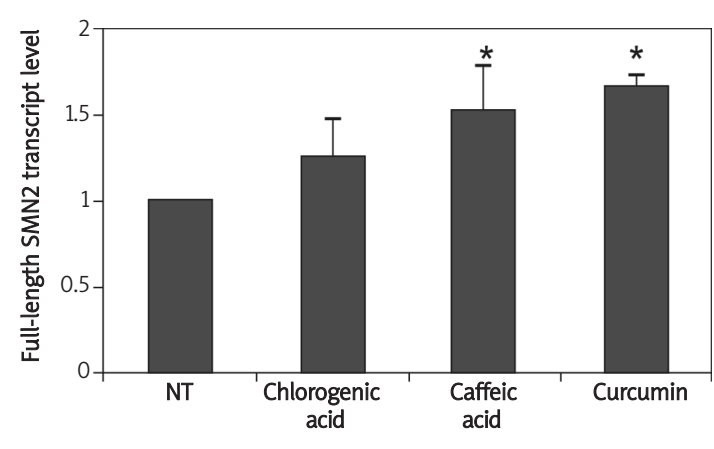

Figure 1. Effect of carboxylic acid derivatives on FL-SMN2 transcript level. SMA fibroblast cell line was treated with chlorogenic acid $(50 \mu \mathrm{M})$, caffeic acid $(20 \mu \mathrm{M})$ and curcumin $(20 \mu \mathrm{M})$ for $24 \mathrm{~h}$. Error bars represent standard deviation

${ }^{*} p<0.05$ shows significant difference in treated compared to untreated
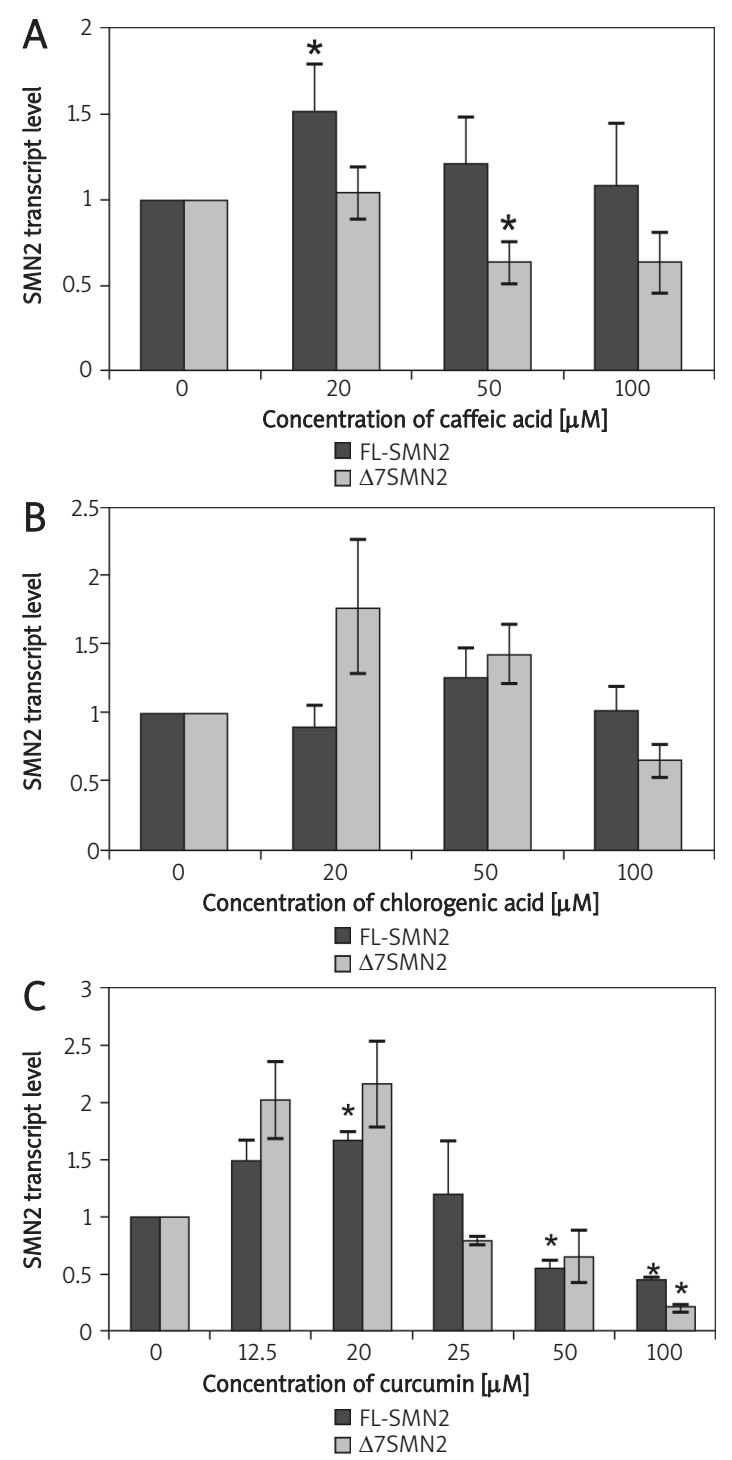

Figure 2. Real-time RT-PCR analysis of FL and $\triangle 7 S M N 2$ transcripts following treatment with caffeic acid $(\mathrm{A})$, chlorogenic acid (B), curcumin (C) at increasing concentrations for $24 \mathrm{~h}$. Error bars represent standard deviation

${ }^{*} p<0.05$ shows significant difference in treated compared to untreated
(Figure 2B). Interestingly, curcumin, which showed a 1.7-fold significant increase in FL-SMN2 mRNA levels, was regarded as the most efficient among all three derivatives (Figure 2C).

To investigate whether caffeic acid, chlorogenic acid and curcumin influence alternative splicing of exon 7, we also studied the effect of three compounds on the synthesis of $\triangle 7 S M N 2$ mRNA levels. In experiments with caffeic acid, no increase in $\triangle 7 S M N 2$ mRNA levels were observed. The ratio of FL to $\Delta 7 \mathrm{mRNA}$ levels increased by almost 2-fold between $0 \mu M$ and $50 \mu M$ (Figure 2A). The treatment with chlorogenic acid resulted in an increase in only $\triangle 7 S M N 2$ mRNA levels, suggesting that chlorogenic acid may work through a different mechanism for SMN2 exon 7 inclusion (Figure 2B). We found that curcumin increased both $\mathrm{FL}$ and $\triangle 7 S M N 2$ mRNA levels at $12.5 \mu \mathrm{M}$ and $20 \mu \mathrm{M}$. The $\mathrm{FL} / \triangle 7$ mRNA level remained constant between $12.5 \mu \mathrm{M}$ and $20 \mu \mathrm{M}$ but increased between $50 \mu \mathrm{M}$ and $100 \mu \mathrm{M}$ (Figure 2C).

We performed an LDH cytotoxicity assay to analyse whether SMN2 activating concentrations (20-50 $\mu \mathrm{M})$ of caffeic acid, chlorogenic acid and curcumin were cytotoxic to fibroblasts. The absorbance of the samples is shown in Figure 3. We found that cytotoxic effects were not detectable and cell viability was $100 \%$.

\section{Discussion}

Recently HDAC inhibitors have been shown to play a role in the treatment of some genetic diseases and also have neuroprotective properties [25]. Therefore they are regarded as candidates for neurodegenerative diseases such as SMA. The disease severity is changed according to functional FL mRNA produced from the SMN2 gene. Therefore increasing SMN2 gene expression has an important role in therapy. The HDAC inhibitors could increase SMN2 gene expression either by directly increasing acetylation at the promoter or changing the

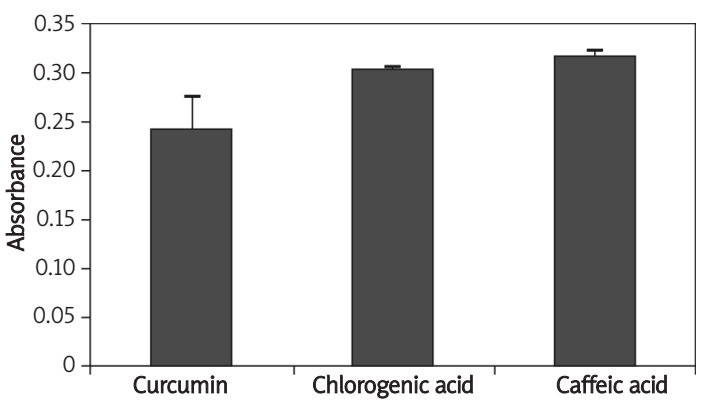

Figure 3. LDH cytotoxicity assay of chlorogenic acid $(50 \mu \mathrm{M})$, caffeic acid $(20 \mu \mathrm{M})$ and curcumin $(20 \mu \mathrm{M})$ on SMA fibroblast cell line. Cytotoxicity was calculated at SMN2 activating concentrations according to the manufacturer's protocols. Low control: 0.380 , high control: 1.87. Error bars represent standard error 
acetylation of a transcription factor that indirectly binds the promoter. Alternatively, HDAC inhibition could change the expression of splicing proteins that increase exon 7 inclusion [12].

The HDAC inhibitors have a common pharmacophore which consists of three components. The metal-binding functional group coordinates binding to the $\mathrm{Zn}^{++}$. The hydrocarbon linker fills out the narrow tunnel, and the capping group interacts with the amino acids near the active site [19]. The design of the new inhibitors is based on the modification of the functional group, capping group and the linker. Carboxylic acid derivatives were modified by insertion of hydroxyl and/or carboxylic groups into the alkyl chain or phenyl ring. As we reported previously, after screening a panel of carboxylic acid derivatives, caffeic acid, chlorogenic acid and curcumin were shown to have the highest HDAC inhibition activity [22]. In our current study, we analysed whether these three compounds produced increased amounts of FL-SMN2 transcript.

Caffeic acid is a polyphenol that occurs in fruits, wine and coffee. It has been shown to have antioxidant, antiinflammatory, antimetastatic and anti-tumour effects [26]. Real-time RT-PCR results showed that FL transcript levels were increased 1.5-fold after caffeic acid treatment. Previous studies indicated that among carboxylic acids, phenylbutyrate and valproic acid increased FL transcript levels between 1.3-1.6 and 1.2-1.5-fold, respectively, in SMA fibroblasts, which is consistent with our data [11-13]. We also found that $\triangle 7 S M N 2$ transcript levels were reduced and the ratio of $\mathrm{FL}$ to $\triangle 7 \mathrm{mRNA}$ levels was increased at some concentrations. The increase in $\mathrm{FL}$ to $\Delta 7$ ratio indicates that caffeic acid may reverse the SMN2 splicing pattern and prevent exon 7 skipping. Enhanced expression of splicing factors that stimulate exon 7 inclusion could be an explanation for this reversion.

By keeping the 4-vinylbenzene-1,2-diol main structure of caffeic acid, two caffeic acid derivatives were chosen. The first derivative, which is chlorogenic acid, is an ester of caffeic acid. We detected a 1.3-fold minor increase in FL-SMN2 transcript levels, which was not statistically significant. It may be speculated that the loss of activity is probably the reason for steric hindrance of the polyfunctional cyclohexane ring.

The second derivative, curcumin, the twin structure of caffeic acid, is found in the Indian curry spice turmeric and shows anti-inflammatory and antioxidant activities [27, 28]. It has been shown that curcumin inhibits proliferation of cells and induces cell cycle arrest or apoptosis in various types of cancers [29]. Cell signal pathways and protein kinases including NF- $\kappa \mathrm{B}, \mathrm{AKT}$, mitogenactivated protein kinase, STAT, COX-2 and LOX have been reported as targets for curcumin [30-32]. The mechanism of curcumin in SMA treatment has not been determined but studies showed that multiple activities of curcumin are involved in the neuroprotective effect of the compound [33]. Following treatment, we found that curcumin showed the highest increase in FL-SMN2 transcript levels among all compounds, which was detected as 1.7-fold. The reason for increased activity may be symmetry in the curcumin molecule. On the other hand, the level of FL-SMN2 at $50 \mu \mathrm{M}$, and the level of FL-SMN2 and $\triangle 7 S M N 2$ at $100 \mu \mathrm{M}$ were decreased. In the context of enzyme kinetics this decrease may suggest that saturation is achieved gradually between HDAC enzyme and its ligand curcumin.

We conclude that the molecular structure of curcumin is the one best fitting the HDAC enzyme active site. Therefore it may be speculated that two phenolic hydroxyl groups and a free carboxylic acid group are not crucial for good interaction with the HDAC enzyme since caffeic acid and chlorogenic acid have one of these two groups. But probably a $p$-hydroxy group is necessary for hydrogen bonds and an enon group for reversible electrostatic interactions with HDAC enzymes.

Similar to our results, Sakla et al. [24] also treated GM03813 cell lines with polyphenols and examined them by RT-PCR. They reported that compounds increase exon 7 inclusion of SMN2 transcripts. We also detected an increase in $\triangle 7 S M N 2$ transcript levels and the ratio of FL to $\Delta 7$ mRNA levels remained constant. This increase in both isoforms may be explained by SMN2 promoter activation. However, detecting an increase in FL/ $\Delta 7$ mRNA levels between $50 \mu \mathrm{M}$ and $100 \mu \mathrm{M}$ treatment suggests that curcumin may also prevent exon 7 skipping. Our data show that curcumin increases FL-SMN2 transcript by not only activating the promoter, but also splicing.

On the basis of these observations, further experiments are being planned on caffeic acid and curcumin to investigate their effect on SMN2 protein. Western blot analysis combined with realtime RT-PCR results will provide more information about the mechanism of the compounds' effect on SMN2 expression.

In recent years, phenolic compounds have become popular for protection against degenerative diseases including SMA [33]. The phenolic structure and replication of the molecule, as demonstrated in curcumin, can be one of the strategies to design novel HDAC inhibitors in the treatment of SMA.

In conclusion, in this study, we found that among carboxylic acid derivatives, caffeic acid and curcumin increase the level of FL-SMN2 mRNA in SMA fibroblasts, indicating that they can be potential candidates in the treatment of SMA. 
Future studies will aim for rational design by keeping the phenolic structure and symmetry of HDAC inhibitors.

\section{Acknowledgments}

This work was supported by the Scientific and Technological Research Council of Turkey, Research Project No: $105 \mathrm{G} 014$.

\section{References}

1. Pearn J. Classification of spinal muscular atrophies. Lancet 1980; 1: 919-22.

2. Crawford TO, Pardo CA. The neurobiology of childhood spinal muscular atrophy. Neurobiol Dis 1996; 3: 97-110.

3. Lefebvre S, Burglen L, Reboullet S, et al. Identification and characterization of a spinal muscular atrophy determining gene. Cell 1995; 80: 155-65.

4. Erdem H, Pehlivan S, Topaloglu H, Ozguc M. Deletion analysis in Turkish patients with spinal muscular atrophy. Brain Dev 1999; 21: 86-9.

5. Rodrigues NR, Owen N, Talbot K, Ignatius J, Dubowitz V, Davies KE. Deletions in the survival motor neuron gene on 5 q13 in autosomal recessive spinal muscular atrophy. Hum Mol Genet 1995; 4: 631-4.

6. Liu Q, Dreyfuss G. A novel nuclear structure containing the survival of motor neurons protein. EMBO J 1996; 15: 3555-65.

7. Lorson CL, Hahnen E, Androphy EJ, Wirth B. A single nucleotide in the SMN gene regulates splicing and is responsible for spinal muscular atrophy. Proc Natl Acad Sci USA 1999; 96: 6307-11.

8. Lorson CL, Androphy EJ. An exonic enhancer is required for inclusion of an essential exon in the SMA-determining gene, SMN. Hum Mol Genet 2000; 9: 259-65.

9. Sumner CJ. Therapeutics development for spinal muscular atrophy. NeuroRx 2006; 3: 235-45.

10. Chang JG, Hsieh-Li HM, Jong YJ, Wang NM, Tsai CH, Li H. Treatment of spinal muscular atrophy by sodium butyrate. Proc Natl Acad Sci USA 2001: 98: 9808-13.

11. Andreassi C, Angelozzi C, Tiziano FD, et al. Phenylbutyrate increases SMN expression in vitro: relevance for treatment of spinal muscular atrophy. Eur J Hum Genet 2004; 12: 59-65.

12. Sumner CJ, Huynh TN, Markowitz JA, et al. Valproic acid increases SMN levels in spinal muscular atrophy patient cells. Ann Neurol 2003; 54: 647-54.

13. Brichta L, Hofmann Y, Hahnen E, et al. Valproic acid increases the SMN2 protein level: a well-known drug as a potential therapy for spinal muscular atrophy. Hum Mol Genet 2003; 12: 2481-9.

14. Riessland M, Brichta L, Hahnen E, Wirth B. The benzamide M344, a novel histone deacetylase inhibitor, significantly increases SMN2 RNA? protein levels in spinal muscular atrophy cells. Hum Genet 2006; 120: 101-10.

15. Avila AM, Burnett BG, Taye AA, et al. Trichostatin A increases SMN expression and survival in a mouse model of spinal muscular atrophy. J Clin Invest 2007; 117: 659-71.

16. Hahnen E, Eyupoglu IY, Brichta L, et al. In vitro and ex vivo evaluation of second-generation histone deacetylase inhibitors for the treatment of spinal muscular atrophy. J Neurochem 2006; 98: 193-202.

17. Kelly WK, O'Connor OA, Marks PA. Histone deacetylase inhibitors: from target to clinical trials. Expert Opin Investig Drugs 2002; 11: 1695-713.
18. Xu WS, Parmigiani RB, Marks PA. Histone deacetylase inhibitors: molecular mechanisms of action. Oncogene 2007; 26: 5541-52.

19. Miller TA, Witter DJ, Belvedere SJ. Histone deacetylase inhibitors. J Med Chem 2003; 46: 5097-116.

20. Moradei O, Maroun CR, Paquin I, Vaisburg A. Histone deacetylase inhibitors: latest developments, trends and prospects. Curr Med Chem Anticancer Agents 2005; 5: 529-60.

21. Garbes L, Riessland M, Hölker I, et al. LBH589 induces up to 10 -fold SMN protein levels by several independent mechanisms and is effective even in cells from SMA patients non-responsive to valproate. Hum Mol Genet 2009; 18: 3645-58.

22. Bora-Tatar G, Dayangaç-Erden D, Demir AS, Dalkara S, Yelekçi K, Erdem-Yurter H. Molecular modifications on carboxylic acid derivatives as potent histone deacetylase inhibitors: activity and docking studies. Bioorg Med Chem 2009; 17: 5219-28.

23. Dayangaç-Erden D, Bora G, Ayhan P, et al. Histone deacetylase inhibition activity and molecular docking of (e )-resveratrol: its therapeutic potential in spinal muscular atrophy. Chem Biol Drug Design 2009; 73: 355-64.

24. Sakla MS, Lorson CL. Induction of full-length survival motor neuron by polyphenol botanical compounds. Hum Genet 2008; 122: 635-43.

25. Hahnen E, Hauke J, Tränkle C, Eyüpoglu IY, Wirth B, Blümcke I. Histone deacetylase inhibitors: possible implications for neurodegenerative disorders. Expert Opin Investig Drugs 2008; 17: 169-84.

26. Chung TW, Moon SK, Chang YC, et al. Novel and therapeutic effect of caffeic acid and caffeic acid phenyl ester on hepatocarcinoma cells:complete regression of hepatoma growth and metastasis by dual mechanism. FASEB J 2004; 18: 1670-81.

27. Calabrese V, Bates TE, Mancuso C, et al. Curcumin and the cellular stress response in free radical-related diseases. Mol Nutr Food Res 2008; 52: 1062-73.

28. Karatepe O, Gülçiçek O, Adas G, et al. The use of curcumin in obstructive jaundice. Arch Med Sci 2009; 5: 513-8.

29. Liu H, Chen Y, Cui G, Zhou J. Curcumin, a potent antitumor reagent, is a novel histone deacetylase inhibitor regulating B-NHL cell line Raji proliferation. Acta Pharmacol Sin 2005; 26: 603-9.

30. Chen Y, Shu W, Chen W, Wu Q, Liu H, Cui G. Curcumin, both histone deacetylase and p300/CBP-specific inhibitor, represses the activity of nuclear factor kappa B and Notch 1 in Raji cells. Basic Clin Pharmacol Toxicol 2007; 101: 427-33.

31. Hong J, Bose M, Ju J, et al. Modulation of arachidonic acid metabolism by curcumin and related beta-diketone derivatives: effects on cytosolic phospholipase A2, cyclooxygenases and 5-lipoxygenase. Carcinogenesis 2004; 25: 1671-9.

32. Liacini A, Sylvester J, Li WQ, Zafarullah M. Inhibition of interleukin-1-stimulated MAP kinases, activating protein-1 (AP-1) and nuclear factor kappa B (NF-kappaB) transcription factors downregulates matrix metalloproteinase gene expression in articular chondrocytes. Matrix Biol 2002; 21: 251-62.

33. Ramassamy C. Emerging role of polyphenolic compounds in the treatment of neurodegenerative diseases: a review of their intracellular targets. Eur J Pharmacol 2006; 545: 51-64. 